\title{
INVESTIGATION OF COLOR REPRODUCTION ACCURACY OF DIFFERENT INK JET AND ELECTROPHOTOGRAPHICAL PRINTING SYSTEMS
}

\author{
Rumyana Boeva (D), Iskren Spiridonov (D), Simeon Yordanov (D), \\ Tatyana Bozhkova (D), Zhivko Ivanov (iD) \\ University of Chemical Technology and Metallurgy, \\ Department of Pulp, Paper and Printing Arts, Sofia, Bulgaria
}

\begin{abstract}
The main goal of this paper is to define and compare the reproduction accuracy and color quality of different digital printing systems. The chosen digital printing systems are from both commonly used types - ink jet and electrophotography. The printers used for experiment in this study are: HP Indigo 5500, Canon IPF 9400, HP Latex 370, Agfa Anapurna M2540 FB. The media used are: glossy and matt coated papers, uncoated papers, photo paper, Jet coat paper, Polypropylene, PVC, vinyl. On above mentioned digital printing systems and media have been printed several test forms with big number of test charts and different control elements. The test forms are containing the following control elements: 240 Pantone colors for assessment of color difference from the original Pantone colors, color charts for generating of ICC profiles, 3D volume and 2D color gamut for different media and printing systems, charts for measuring of color characteristics of main and secondary colors, charts for evaluation of tone value increase, etc. A big number of experimental results are obtained for the most important printing quality parameters color difference from original Pantone colors, color gamut volume, accuracy of reproduction expressed by tone values, etc.
\end{abstract}

Key words: printing quality, color reproduction accuracy, digital printing

\section{INTRODUCTION}

During the past few years the main driving force of technical development is established by digital technology, which advances ever so greatly in all scientific domains, in economy and in industry. The new generation machines, equipment, systems abd materials contribute to improved performance, better quality, greater ecology and cost efficiency. New printing technologies offer reductions in the use of materials and energy, as well as shorter machine setup time and decrease of maculatures. In this context, the development of graphic technologies is a stive to ameliorate the efficiency of atleast one of the three main principles of every business, namely - rapidity, quality and production costs.

Digital print is a new generation technology in comparison to all known methods of printing information on paperback or any other type of carrier. With the development of information technologies, the application of this printing method increases as well. It picks up steal in the Graphic industry, as the tendency is for it to go into full industrial use for the production of packages and labels in small and medium circulations (Kachin et al, 2004, Kachin et al, 2000). This paper is the first part of a series of researches, which are focused on different classes of digital printing machines as the latter are used in a variety of applications, such as commercial Printing, outdoors advertisement, Packaging and Labels, etc.

\section{EXPERIMENTAL}

Reaching the set goals was aided by the use of different measurement tools - densitometer, spectrophotometer, which served to examine the accuracy and quality of tonal and colour reproduction in digital printing machines. In order to maintain result reproducibility, a specialised test form was developed and used. The test forms consisits of:

- $\quad$ ECl 2002 test chart with 1485 patches for colorimetric assessment and generating of ICC profiles;

- $\quad$ Test charts with 0, 5, 10, 20 to 100\% for all process colors and their double overlays;

- Positive and negative lines of different width and number of colors for assessment in order to detect the thinnest lines, which could be reproduced from the given material by the examined printing systems;

- Test images for visual analyses;

- $\quad$ Small positive and negative text of different size;

- Vighetes. 
A multitude of colorimetry and densitometry methods were used. The goal of this paper is to render examination results on the accuracy and quality of tomal and colour reproduction in ink-jet and electrophotographical digital print as a multitude of assessment methods related to tonal and colour reproduction accuracy were selected regarding the Pantone colouring system, colour characteristics of solid primary colors, tone value increase, assessment of $3 \mathrm{~d}$ and $2 \mathrm{~d}$ color gamut shape and values, visual analyses of images, reproduction of thin positive and negative line art and lines, as well as fonts with small size in one, two and more colours (Kachin, 2004; Kipphan, 2001). The following digital machines have been used to carry out the experiment:

- $\quad$ Agfa Anapurna M 2540FB - ink-jet printer with UV inks;

- $\quad$ Canon IPF 9400 - ink-jet printer with water-based inks;

- HP Latex 370 - ink-jet printer with water-based inks;

- HP Indigo 5500 - electrophotographical printer with toner inks.

Used media to perform the experimental analyses are:

- $\quad$ Offset uncoated paper $-120 \mathrm{~g} / \mathrm{m}^{2}$;

- Matte Coated paper- $150 \mathrm{~g} / \mathrm{m}^{2}$;

- Glossy Coated paper $-150 \mathrm{~g} / \mathrm{m}^{2}$;

- Paper "Jetcoat" - $200 \mathrm{~g} / \mathrm{m}^{2}$;

- $\quad$ Glossy Photo paper - $190 \mathrm{~g} / \mathrm{m}^{2}$;

- Polypropylene $-160 \mathrm{~g} / \mathrm{m}^{2}$;

- $\quad$ Self-adhesive PVC - glossy $-135 \mathrm{~g} / \mathrm{m}^{2}$;

- $\quad$ Vinyl $-440 \mathrm{~g} / \mathrm{m}^{2}$.

\section{RESULTS AND DISCUSSION}

Due to the large dataset obtained from the thorough research on the precision and quality of tonal and colour reproduction for ink-jet and electrophotographical digital print, this paper will only include the results, obtained during the experiments with HP Indigo 5500, loaded with offset uncoated paper and glossy coated. The examination included assessment of colour characteristics of test prints, simulating specially selected Pantone colours. Also there is a measurement of the colour coordinates as per the CIE Lab system with included calculation and comparison of $\Delta \mathrm{E}_{76}$ and $\Delta \mathrm{E}_{00}$ according to (ISO 12647-2:2013). A dedicated software product was used to render the $2 \mathrm{D}$ and $3 \mathrm{D}$ visualisation and a comparison between the colour gammuts of the examined media was made with a standard ICC profile FOGRA47 and FOGRA51.

In order to compare the quality of tonal and colour reproduction of catalogue and selected Pantone system colours, one must calculate the colour difference $\Delta \mathrm{E}$ for a set number of colours. In order to calculate $\Delta \mathrm{E}$, one must first obtain the colour coordinates of all colours according to the CIE Lab system. The reference values are the colour coordinates as per (ISO 12647-2:2013).

Process control for the production of half-tone colour Colour differences are derived using the following formulas:

- $\quad$ colour difference $\Delta \mathrm{E}_{76}$ :

$$
\Delta E_{76}^{*}=\sqrt{\left(L_{1}^{*}-L_{2}^{*}\right)^{2}+\left(a_{1}^{*}-a_{2}^{*}\right)^{2}+\left(b_{1}^{*}-b_{2}^{*}\right)^{2}}
$$

- $\quad$ colour difference $\Delta \mathrm{E}_{00}$ :

$$
\Delta E_{00}^{*}=\sqrt{\left(\frac{\Delta L^{\prime}}{K_{L} S_{L}}\right)^{2}+\left(\frac{\Delta C^{\prime}}{K_{C} S_{C}}\right)^{2}+\left(\frac{\Delta H^{\prime}}{K_{H} S_{H}}\right)^{2}+R_{T} \frac{\Delta C^{\prime}}{K_{C} S_{C}} \frac{\Delta H^{\prime}}{K_{H} S_{H}}}
$$

Where: $\quad \mathrm{L}-$ lightness/brightness

$\mathrm{C}$ - saturation

$\mathrm{H}$ - hue 
The measurements of colour coordinates are made using a spectrophotometer under the following conditions:

- $\quad$ Standard light illuminant - D50;

- $\quad 2^{\circ}$ standard observer;

- No polarisation filter;

- Aperture-4mm;

- A white backing has been used.

\subsection{Investigation of color reproduction accuracy of digital printing systems and calculation of $\Delta \mathrm{E}$ for Pantone colours}

In order to examine the reproduction precision of the fundamental Pantone colours, the colour coordinates (Lab) for the media listed above have been measured. Figure 1 represents the basic Pantone colours.
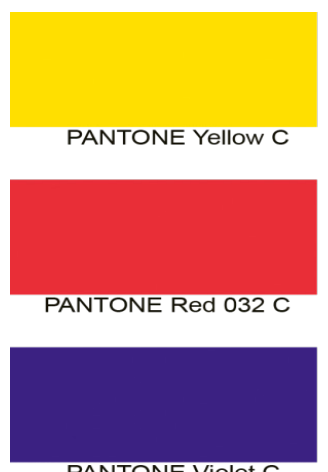

PANTONE Violet C

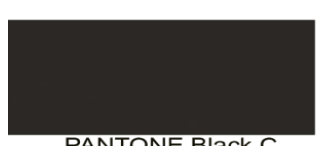

PANTONE Black C
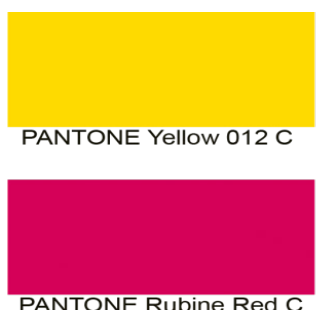

PANTONE Rubine Red C

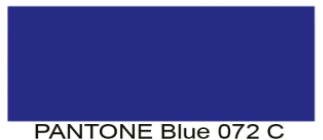

PANTONE Blue $072 \mathrm{C}$
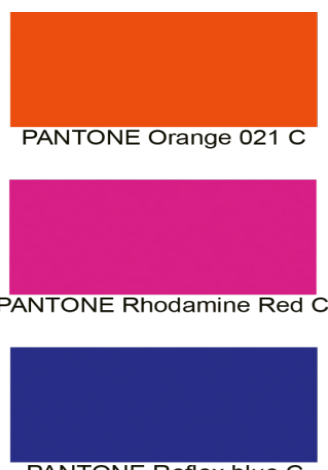

PANTONE Reflex blue C
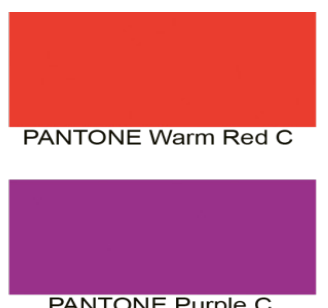

PANTONE Purple C

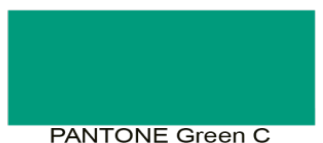

Figure 1: Basic Pantone colours used in experiment

In order to examine the reproduction precision of the basic Pantone colours, the colour coordinates (Lab) for the media listed above have been measured. Figure 43 represebts the basic Pantone colours. Figure 2 represents a selection of Pantone colours.

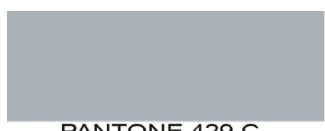

PANTONE 429 C

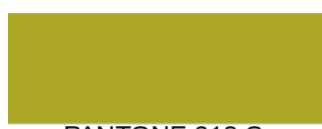

PANTONE $618 \mathrm{C}$

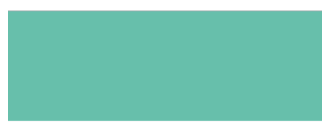

PANTONE $7465 \mathrm{C}$

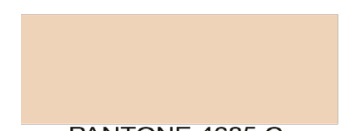

PANTONE $4685 \mathrm{C}$

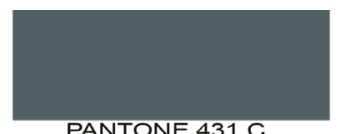

PANTONE $431 \mathrm{C}$

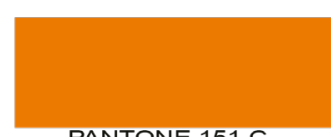

PANTONE $151 \mathrm{C}$

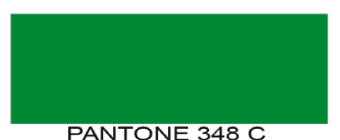

PANTONE $348 \mathrm{C}$

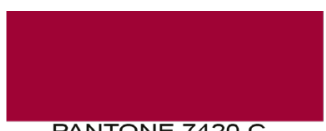

PANTONE $7420 \mathrm{C}$

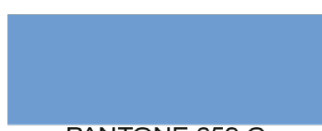

PANTONE 659 C

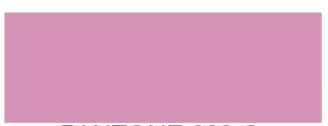

PANTONE 6800

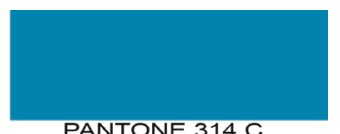

PANTONE 314 C

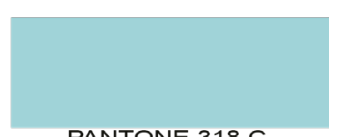

PANTONE $318 \mathrm{C}$

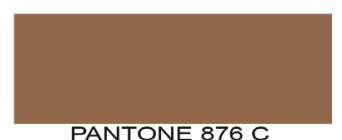

Figure 2: Selection of Pantone colours used in experiment 


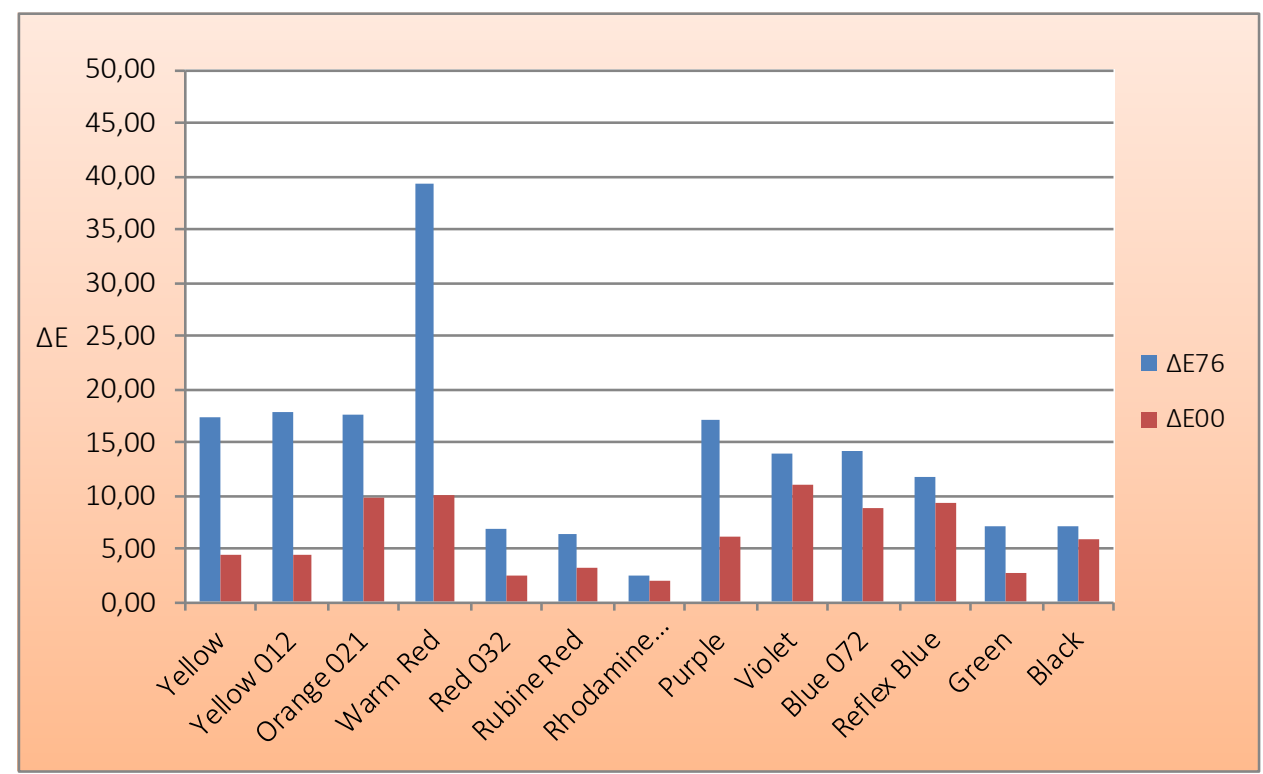

Figure 3: Comparison of the colour differences, according to CIE Lab, between the basic Pantone colours with $\Delta E_{76}$ and $\Delta E_{00}$ for Offset uncoated paper printed through HP Indigo 5500

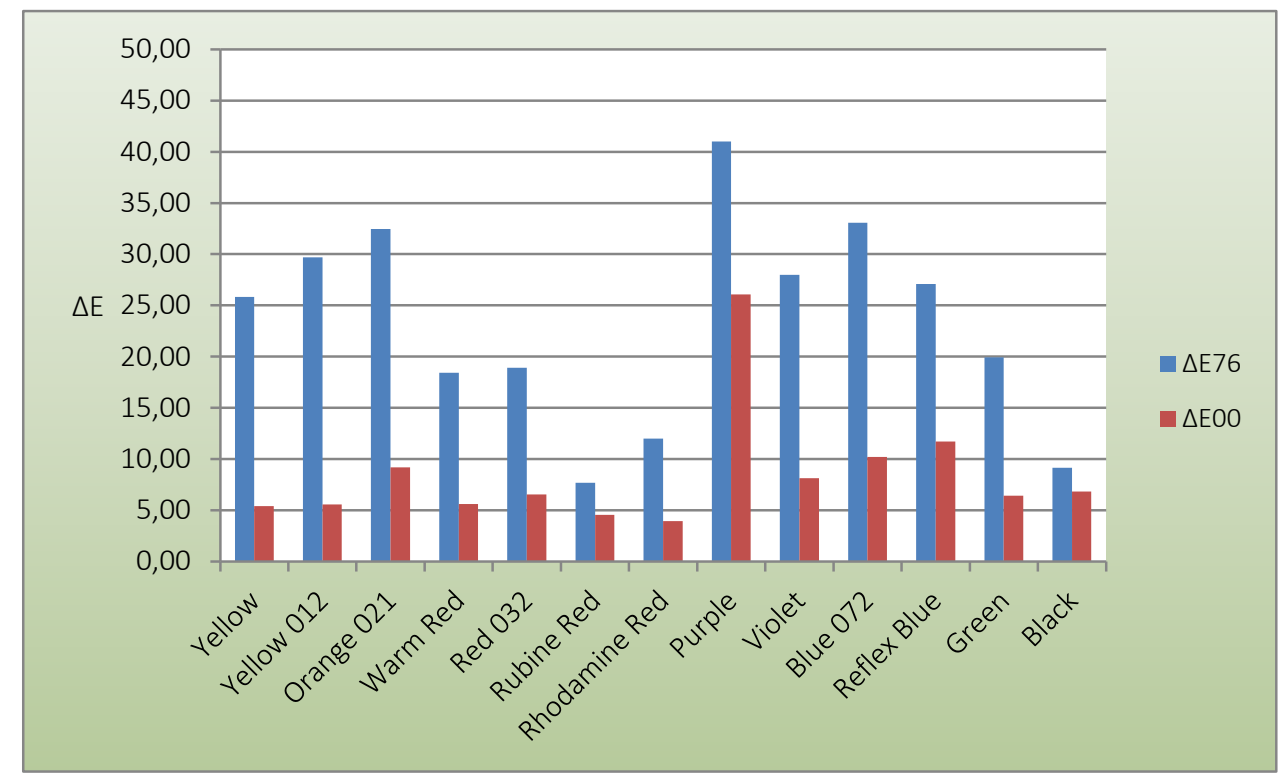

Figure 4: Comparison of the colour differences, according to CIE Lab, between the basic Pantone colours with $\Delta E_{76}$ and $\Delta E_{00}$ for Glossy Coated paper printed through HP Indigo 5500

Figure 3 and Figure4 shows the comparative graphs for comparison of the colour differences, according to $\mathrm{CIE} L a b$, between the basic Pantone colours with $\triangle \mathrm{E} 76$ and $\triangle \mathrm{E} 00$ for offset uncoated paper printed through HP Indigo 5500 and comparison of the colour differences, according to CIE Lab, between the basic Pantone colours with $\triangle \mathrm{E} 76$ and $\triangle \mathrm{E} 00$ for Glossy Coated paper printed through HP Indigo 5500.

Due to the extensiveness of the measurements and the multitude of obtained results, this paper presents only an excerpt there to concerning the stated printing machine and the printing media, used in conjunction with it. Tables 1 and 2 show all results derived from $\Delta \mathrm{E}$ calculations. 
Table 1: Experimental results for color reproduction accuracy obtained for basic Pantone colours

\begin{tabular}{|c|c|c|c|c|c|c|c|c|c|c|c|c|c|c|}
\hline \multicolumn{3}{|c|}{ Fundamental Pantone colours } & \multicolumn{3}{|c|}{$\Delta \mathrm{E} 76$} & \multicolumn{3}{|c|}{$\begin{array}{l}\text { Count of colours corresponding to the } \\
\text { average limitations (total of 13) }\end{array}$} & \multicolumn{3}{|c|}{$\Delta \mathrm{E} 00$} & \multicolumn{3}{|c|}{$\begin{array}{c}\text { Count of colours corresponding to the } \\
\text { average limitations (total of 13) }\end{array}$} \\
\hline № & Digital machine & Media & $\Delta E$ avg. & $\Delta E \min$ & $\Delta \mathrm{Emax}$ & $\Delta E<3$ & $\Delta E<5$ & $\Delta E>5$ & $\Delta \mathrm{E}$ avg. & $\Delta E \min$ & $\Delta E \max$ & $\Delta E<3$ & $\Delta E<5$ & $\Delta E>5$ \\
\hline 1 & HP Indigo 5500 & Offset uncoated paper & 13,77 & 2,40 & 39,23 & 1 & 0 & 12 & 6,17 & 2,00 & 11,06 & 3 & 3 & 7 \\
\hline 2 & HP Indigo 5500 & Matte coated paper & 22,59 & 5,69 & 44,13 & 0 & 0 & 13 & 7,04 & 2,56 & 11,70 & 1 & 1 & 11 \\
\hline 3 & HP Indigo 5500 & Glossy coated paper & 23,33 & 7,95 & 33,84 & 0 & 0 & 13 & 7,06 & 4,10 & 11,33 & 0 & 3 & 10 \\
\hline 4 & HP Latex 370 & Self-adhesive PVC & 25,16 & 5,23 & 45,05 & 0 & 0 & 13 & 7,20 & 3,00 & 12,23 & 1 & 1 & 11 \\
\hline 5 & Canon IPF9400 & Photo paper & 25,37 & 5,57 & 46,77 & 0 & 0 & 13 & 7,40 & 2,93 & 12,62 & 1 & 1 & 11 \\
\hline 6 & HP Latex 370 & Jetcoat paper & 25,50 & 5,95 & 43,98 & 0 & 0 & 13 & 7,45 & 3,91 & 12,20 & 0 & 2 & 11 \\
\hline 7 & Agfa Anapurna M2540 & Vinil & 25,87 & 7,66 & 45,88 & 0 & 0 & 13 & 8,26 & 3,65 & 12,09 & 0 & 2 & 11 \\
\hline 8 & HP Latex 370 & polypropylene & 25,98 & 7,70 & 41,01 & 0 & 0 & 13 & 8,48 & 3,95 & 26,06 & 0 & 2 & 11 \\
\hline
\end{tabular}

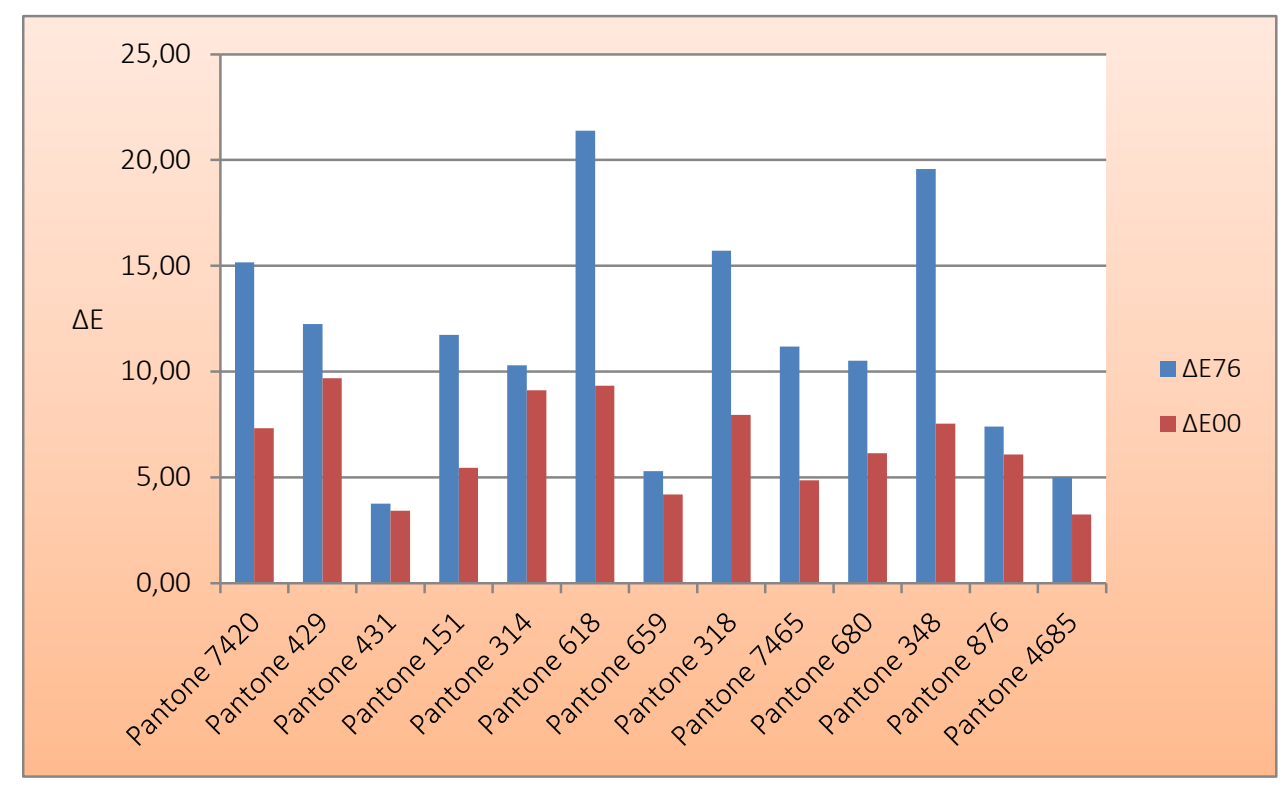

Figure 5: Comparison of the colour differences, according to CIE Lab, between the selected Pantone colours with $\Delta E_{76}$ and $\Delta E_{00}$ for Offset uncoated paper printed through HP Indigo 5500

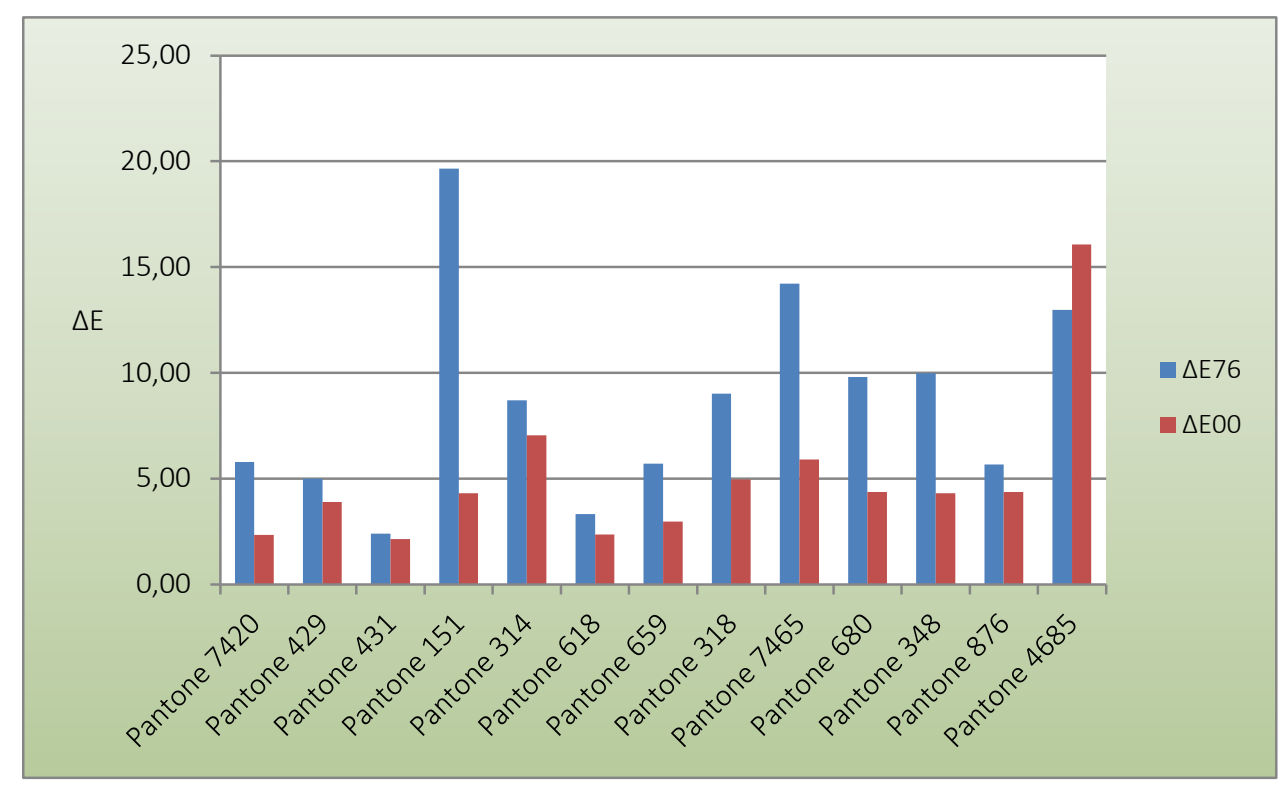

Figure 6: Comparison of the colour differences, according to CIE Lab, between the selected Pantone colours with $\Delta E_{76}$ and $\Delta E_{00}$ for Glossy Coated paper through HP Indigo 5500 
Figure 5 and Figure 6 shows the comparative graphs for comparison of the colour differences, according to CIE Lab, between the selected Pantone colours with $\triangle \mathrm{E} 76$ and $\triangle \mathrm{E} 00$ for Offset uncoated paper printed through HP Indigo 5500 and comparison of the colour differences, according to CIE Lab, between the selected Pantone colours with $\triangle \mathrm{E} 76$ and $\triangle \mathrm{E} 00$ for Glossy Coated paper through HP Indigo 5500

Table 2: Experimental results for color reproduction accuracy obtained for selected Pantone colours

\begin{tabular}{|c|c|c|c|c|c|c|c|c|c|c|c|c|c|c|}
\hline \multicolumn{3}{|c|}{ Selected Pantone colours } & \multicolumn{3}{|c|}{$\Delta \mathrm{E} 76$} & \multicolumn{3}{|c|}{$\begin{array}{c}\text { Count of colours corresponding to the } \\
\text { average limitations (total of 13) }\end{array}$} & \multicolumn{3}{|c|}{$\Delta E 00$} & \multicolumn{3}{|c|}{$\begin{array}{l}\text { Count of colours corresponding to the } \\
\text { average limitations (total of 13) }\end{array}$} \\
\hline № & Digital machine & Media & $\Delta \mathrm{E}$ avg. & $\Delta \mathrm{Emin}$ & $\Delta E \max$ & $\Delta \mathrm{E}<3$ & $\Delta \mathrm{E}<5$ & $\Delta E>5$ & $\Delta E$ avg. & $\Delta \mathrm{Emin}$ & $\Delta E \max$ & $\Delta E<3$ & $\Delta \mathrm{E}<5$ & $\Delta E>5$ \\
\hline 1 & HP Latex 370 & polypropylene & 7,16 & 1,42 & 23,94 & 2 & 4 & 7 & 3,30 & 0,48 & 6,21 & 7 & 3 & 3 \\
\hline 2 & HP Latex 370 & Self-adhesive PVC & 7,21 & 2,31 & 24,17 & 2 & 3 & 8 & 3,36 & 1,25 & 6,40 & 8 & 2 & 2 \\
\hline 3 & Agfa Anapurna M2540 & Vinil & 7,34 & 2,31 & 22,03 & 3 & 3 & 7 & 3,44 & 1,43 & 8,47 & 8 & 2 & 3 \\
\hline 4 & HP Latex 370 & Jetcoat paper & 7,44 & 2,03 & 24,30 & 2 & 3 & 8 & 3,48 & 1,18 & 7,44 & 7 & 3 & 3 \\
\hline 5 & HP Indigo 5500 & Matte coated paper & 7,59 & 1,69 & 18,68 & 2 & 2 & 9 & 3,83 & 1,49 & 5,95 & 4 & 7 & 2 \\
\hline 6 & Canon IPF9400 & Photo paper & 8,28 & 3,72 & 21,07 & 0 & 3 & 10 & 4,18 & 1,99 & 7,15 & 5 & 3 & 5 \\
\hline 7 & HP Indigo 5500 & Glossy coated paper & 8,63 & 2,41 & 19,65 & 1 & 1 & 11 & 5,00 & 2,15 & 16,07 & 4 & 7 & 2 \\
\hline 8 & HP Indigo 5500 & Offset uncoated paper & 11,49 & 3,76 & 21,39 & 0 & 1 & 12 & 6,49 & 3,25 & 9,68 & 0 & 4 & 9 \\
\hline
\end{tabular}

\subsection{Investigation of Tone value increase for digital printing systems on different papers}

The tone value increase is an indicator, which defines tone and colour reproduction and under certain conditions it influences the image depth field. This is one of the most important measured values, serving to control the printing process and is directly linked to the quality of the printed image [Kachin, 2004]. In order to define the tone value increase for the separate prints, one performs densitometric measurements of tonal fields in the interval between $5 \%$ and $100 \%$.

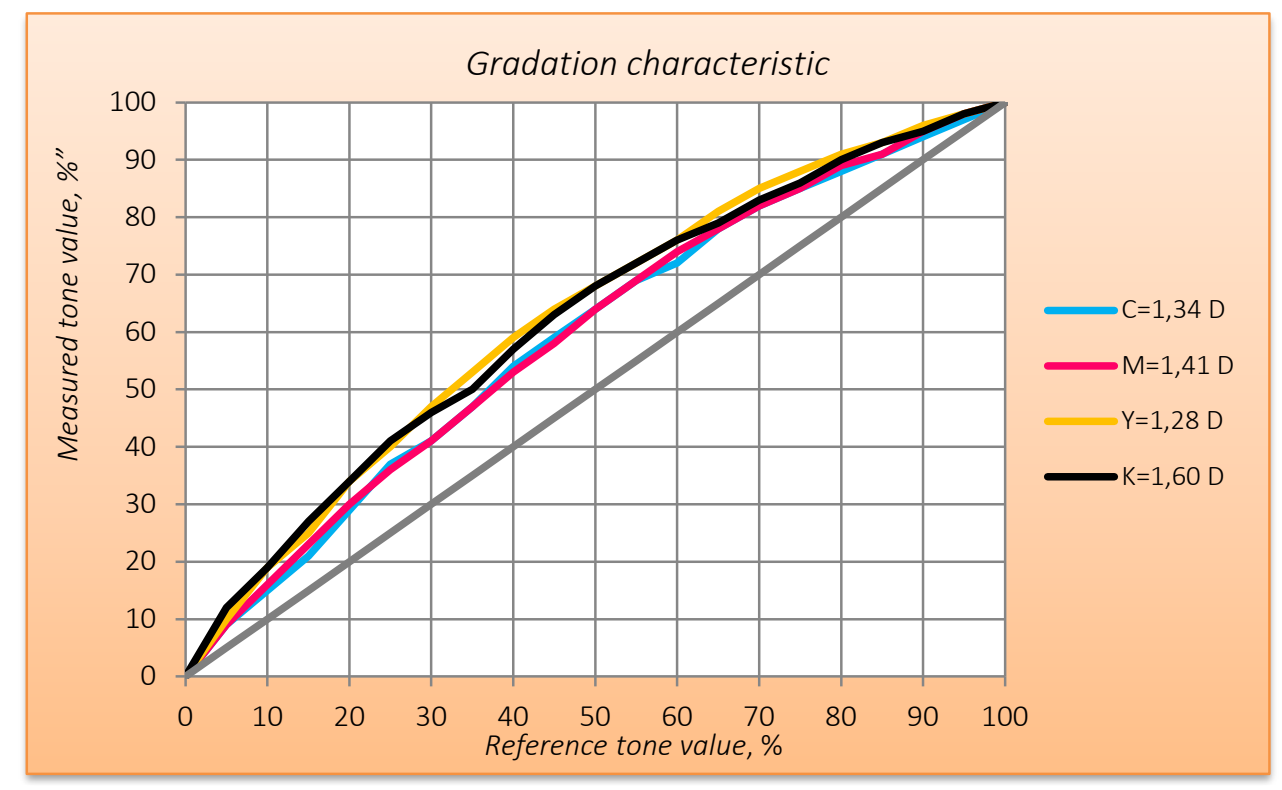

Figure 7: Dependancy between the reference and measured tone value (gradation characteristic) for Offset uncoated paper printed through digital printer HP Indigo 5500

Figure 7 represents the dependency between the set and measured tone value, for offset paper, as this correlation is a gradation characteristic of reproduction for the four primary colours (CMYK). From this figure it is apparent that with the four primary colours (cyan, magenta, yellow and black) we can observe significant uniform increase in the range up to $20 \%$, after which one can observe that with black and yellow there is a slightly higher measured tone value, which varies for both colours in the range between $30 \%$ and $70 \%$. The increase of tone value with black and yellow varies between 4 and 7 units, compared to cyan and magenta. The figure above also demonstrates the extensive similarity of tone values for cyan and magenta across the range. 


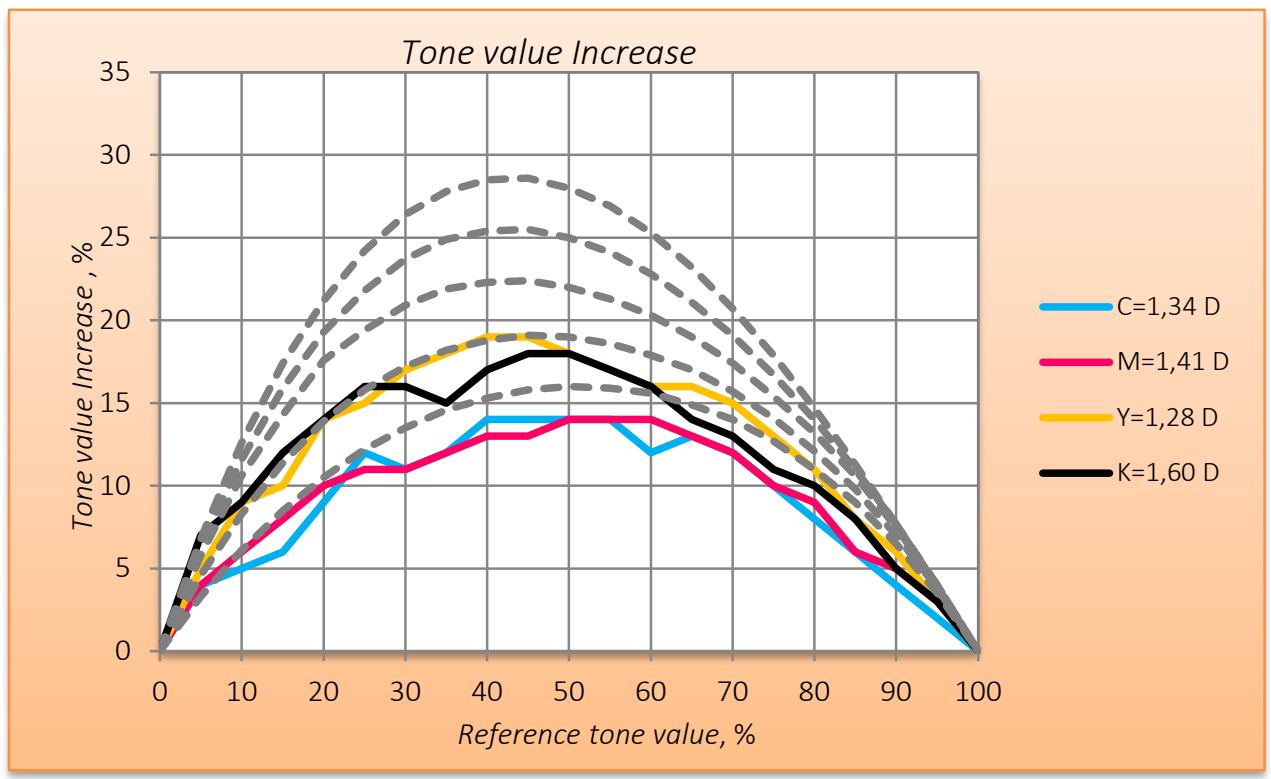

Figure 8: Tone value Increase for the four primary colours and standard curves as per ISO 12647-2:2013 for Offset uncoated paper printed through digital printer HP Indigo 5500

Figure 8 contains the correlation between the reference tone value and the increase of tone value for cyan, magenta, yellow and black, all printed on offset paper, compared to the standard curves as per ISO (ISO 12647-2:2013). The figure shows that cyan shows lower measured values in the range between $5 \%$ and $100 \%$ compared to the reference curves (A-E) as stated in ISO (ISO 12647-2:2013). Magenta shows that in terms of light tones (0\% to 20\%) there is full coincidence with curve A and after the $20 \%$ mark the results are lower than the standard. Yellow displays relatively identical measured values, compared to the reference curve B up to the mid tones, after which it drops down to curve $A$ between $50 \%$ and $70 \%$. The black colour results in solid measurements of tone value increase between $0 \%$ and $30 \%$, after that the values start varying between $30 \%$ and $50 \%$, and in the dark tones, the measurements are lower in pitch than the reference ones. None of the experimental curves shows significant increase of tone value. TVI of cyan and magenta is relatively the same.

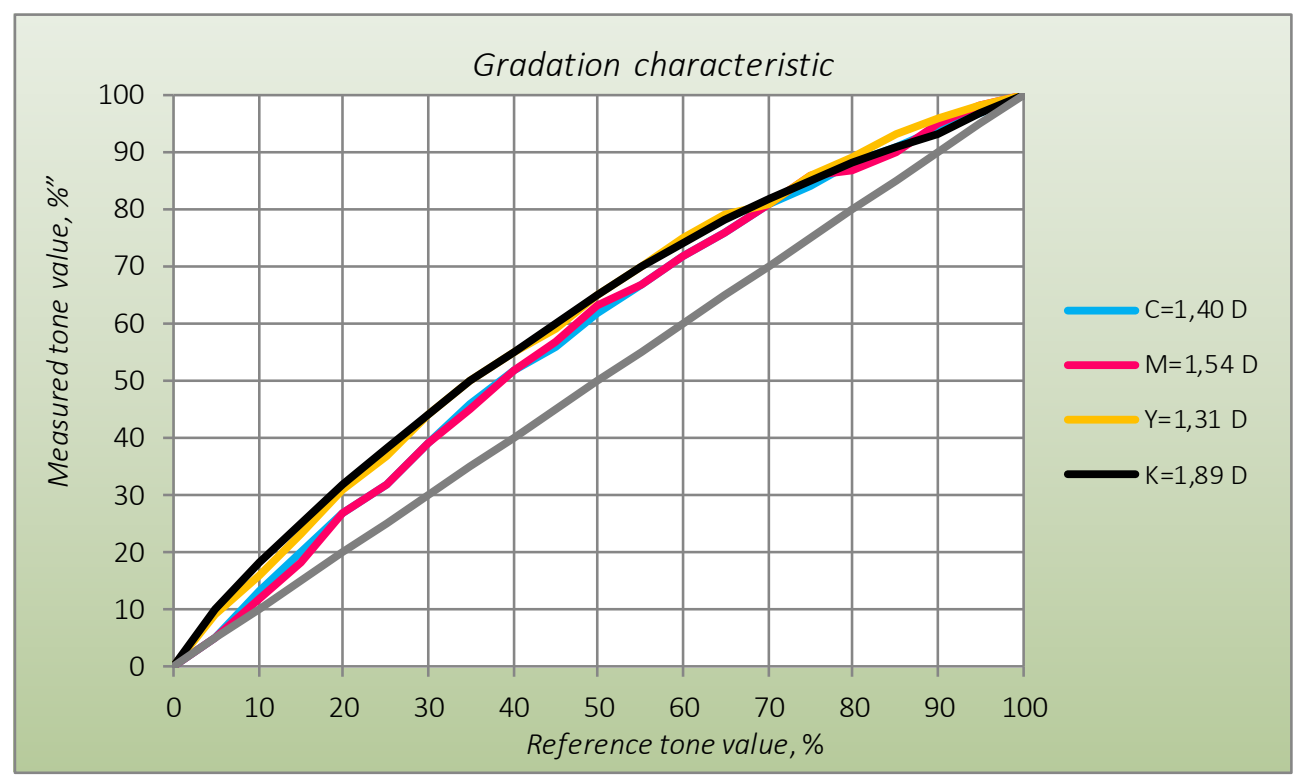

Figure 9: Dependancy between the reference and measured raster tone (gradation characteristic) for gloss paper printed through digital printer HP Indigo 5500 
Figure 9 shows the dependency between the reference and measured tone values, for gloss paper, which represents the gradation characteristic of reproduction for the four primary colours (CMYK). The figure shows that for these four colours (cyan, magenta, yellow and black) one can observe the rather even increase as cyan and magenta almost coincide in the range between $20 \%$ and $70 \%$. Yellow and black have slightly higher values compared to the other two colours.

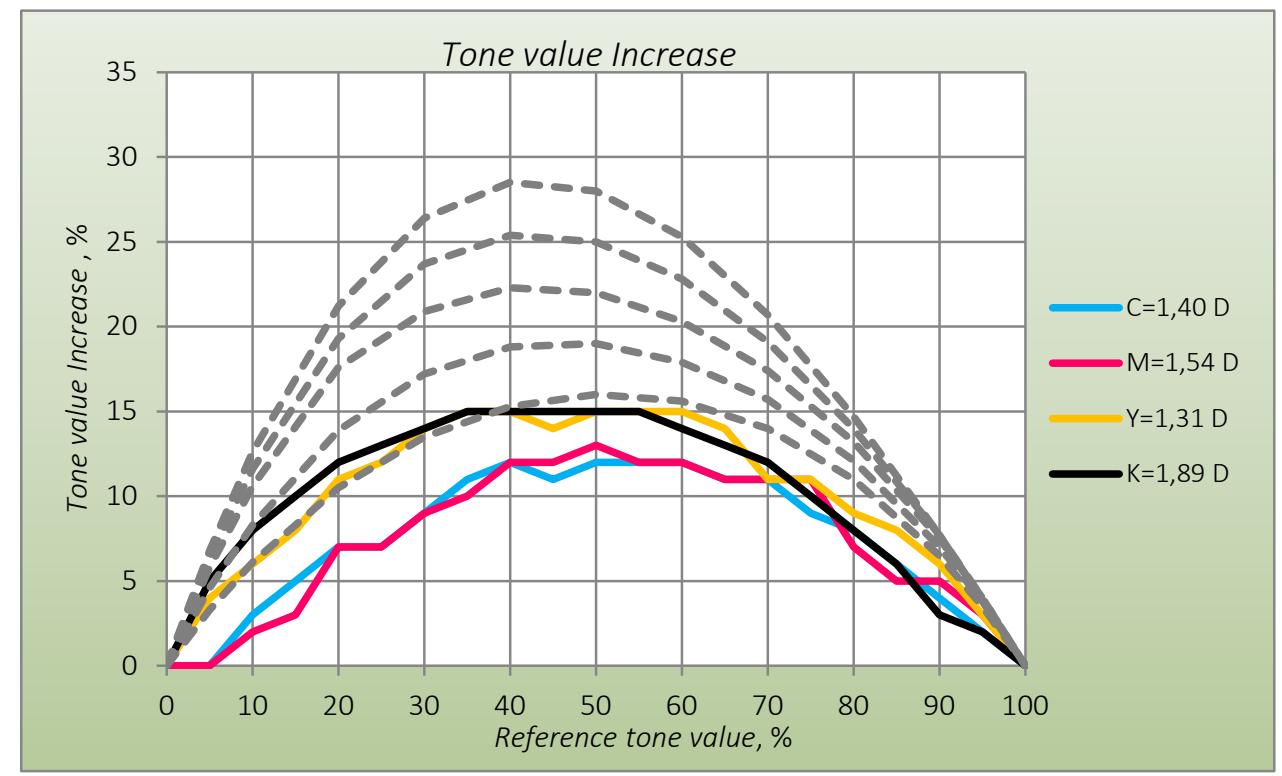

Figure 10: Tone value Increase for the four primary colours and standard curves as per ISO 12647-2:2013 for gloss paper printed through digital printer HP Indigo 5500

Figure 10 shows the correlation between the reference tone value and the increase of tone value for cyan, magenta, yellow and black, all printed on gloss paper, compared to the standard curves as per ISO (ISO 12647-2:2013). The figure shows that the experimental curves of cyan and magenta, for the increase of tone value, are significantly lower in terms of values compared to the reference curves (A-E) as stated in (ISO 12647-2:2013). Both colours (cyan and magenta) are with approximately identical values throughout the test. In the case of the yellow and black colours one observes smoother increase as both colours, in the range $0 \%$ to $40 \%$ follow the reference curves closely and after the mid tones yellow shows slight decrease between $40 \%$ and $50 \%$. This figure does not show a peak increase shift for both light and dark tones due to the fact that the four colours have lower measured values, compared to the references. However, this may also lead to inaccuracy of tonal and colour reproduction. From the cyan and magenta curves, it is clear that there is $0 \%$ increase in the first $5 \%$ of the reference tone value.

\subsection{Investigation of color gamut volumes and comparison of 2D and 3D color gammuts of different digital printing systems}

One important function, which is used to visualise the colours, as reproduced by any given machine, is the $2 \mathrm{D}$ and $3 \mathrm{D}$ representation of the respective colour gammuts. This paper also uses 2D and 3D representations. The 2D representation of colour gammuts with different cross-sections along the L-axis of the CIE Lab colour space allows for good visual comparison of colours in light, mid and dark tones, as well as comparison of a large number of colour gammuts at once. The 3D representation of colour gammuts allows a complex visual assessment for the 3D body of the colour gammut. It is appropriate for the visualisation and comparison of ore or two colour gammuts.

In order to perform the comparison for the colour gammut of the examined prints from the corresponding digital printing presses - HP Indigo 5500, Agfa Anapurna M2540, Canon IPF9400 and HP Latex 370 it is necessary to provide a 3D visualisation with a standard ICC profile FOGRA 47 for uncoated papers and FOGRA 51 for coated papers with the ultimate goal to achieve visual representation for the colour gammuts. The 3D visualisation of the ICC colour profiles was performed using the software PROFILE MAKER 5.10. To compare the colour gammut on offset uncoated paper, a standard ICC profile was used FOGRA 47. 


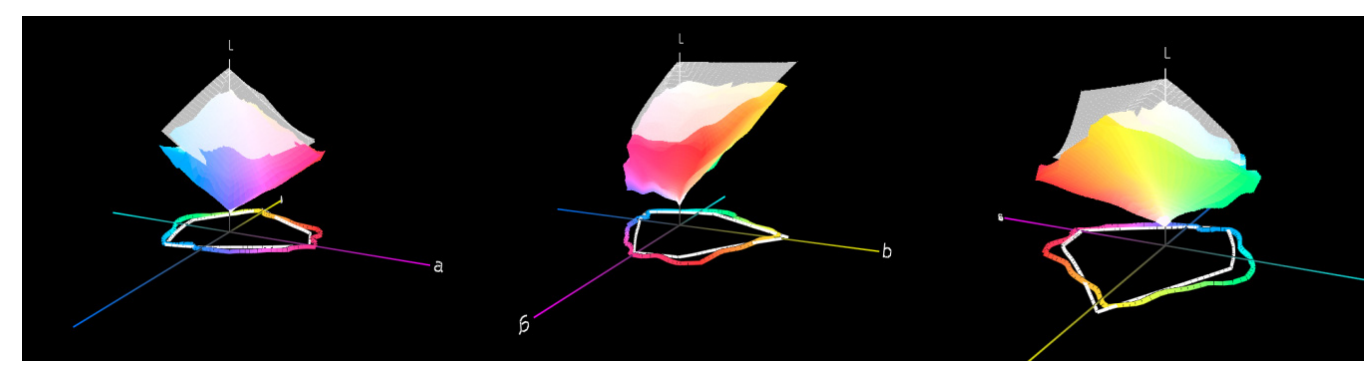

Figure 11: 3D visualisation (Lab system) of an ICC profile of offset paper printed through HP Indigo 5500 and FOGRA 47

From figure 11 one can observe, that the colour gammut of FOGRA47 is significantly larger than the one of the offset paper, which was printed through the HP Indigo 5500. It is also noticeable, that in certain areas the colour gammut of the tested digital machine - material is able to reproduce colours, which FOGRA47 could not [fogra.org, eci.org]. It is also clear that with offset paper one obtains better colour reproduction of the light hues of the yellow-red and the blue-green areas. In FOGRA 47, however, one notices better reproduction of darker hues.

As a comparison platform for the colour gammuts of matte paper, gloss paper, vinil, photo paper, Jetcoat paper, polypropylene and self-adhesive film, the ICC FOGRA 51 profile has been used:

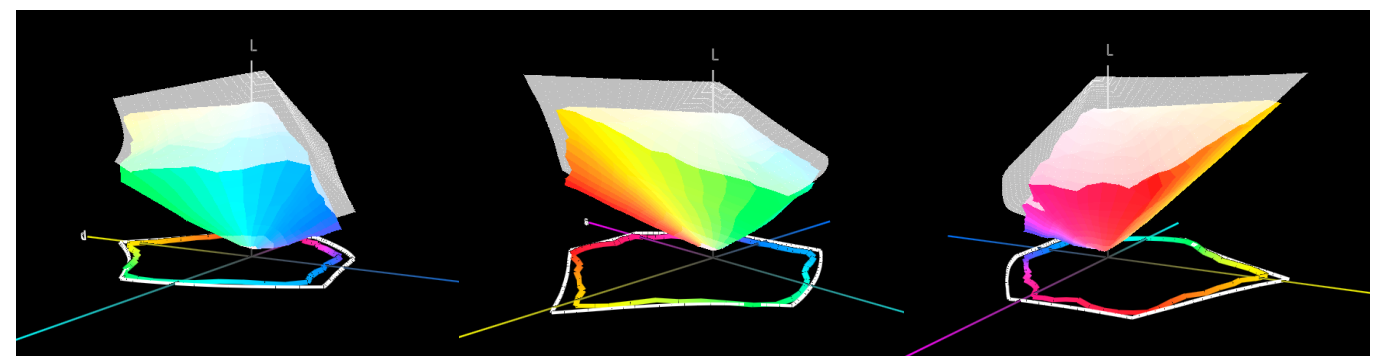

Figure 12: 3D visualisation (Lab system) of an ICC profile of gloss paper printed through HP Indigo 5500 and FOGRA 51

Figure 12 shows that the colour gammut of FOGRA51 is significantly larger compared to the gloss paper printed through the HP Indigo 5500. It is also observed that in certain areas, the colour gammut of the tested digital machine - material is able to reproduce colours, which FOGRA51 cannot. It is also clear that with gloss paper one obtains, once more, better colour reproduction of the light hues of the yellow-red and the blue-green areas. In FOGRA 51, however, one notices better reproduction of darker hues.

Figure 13 depicts a 2D visualisation of the colour gammuts of the tested media. The data shows that the most accurate tone and colour reproduction occurs on matte and gloss papers printed through HP Indigo 5500 , as they coincide in all areas. The smallest colour gammut occurs with offset paper printed through the same machine. This phenomenon is due to the fact, that offset paper is considered an uncoated print media and has more porous structure, thus causing more ink intake in comparison to coated media.

Table 3: Volume of the colour gammut

\begin{tabular}{|c|c|c|c|}
\hline & & & Volume of the colour gammut \\
№ & Machines & Used media & $\Delta E^{3}$ \\
\hline 1 & HP Indigo 5500 & Offset uncoated paper & 202479 \\
\hline 2 & HP Indigo 5500 & Matte Coated paper & 293021 \\
\hline 3 & HP Indigo 5500 & Glossy Coated paper & 314299 \\
\hline 4 & Agfa Anapurna M2540 & Vinil & 244498 \\
\hline 5 & Canon IPF 9400 & Glossy Photo paper & 246780 \\
\hline 6 & HP Latex 370 & Paper “Jetcoat” & 236614 \\
\hline 7 & HP Latex 370 & Polypropylene & 254321 \\
\hline 8 & HP Latex 370 & Self-adhesive PVC & 244983 \\
\hline
\end{tabular}




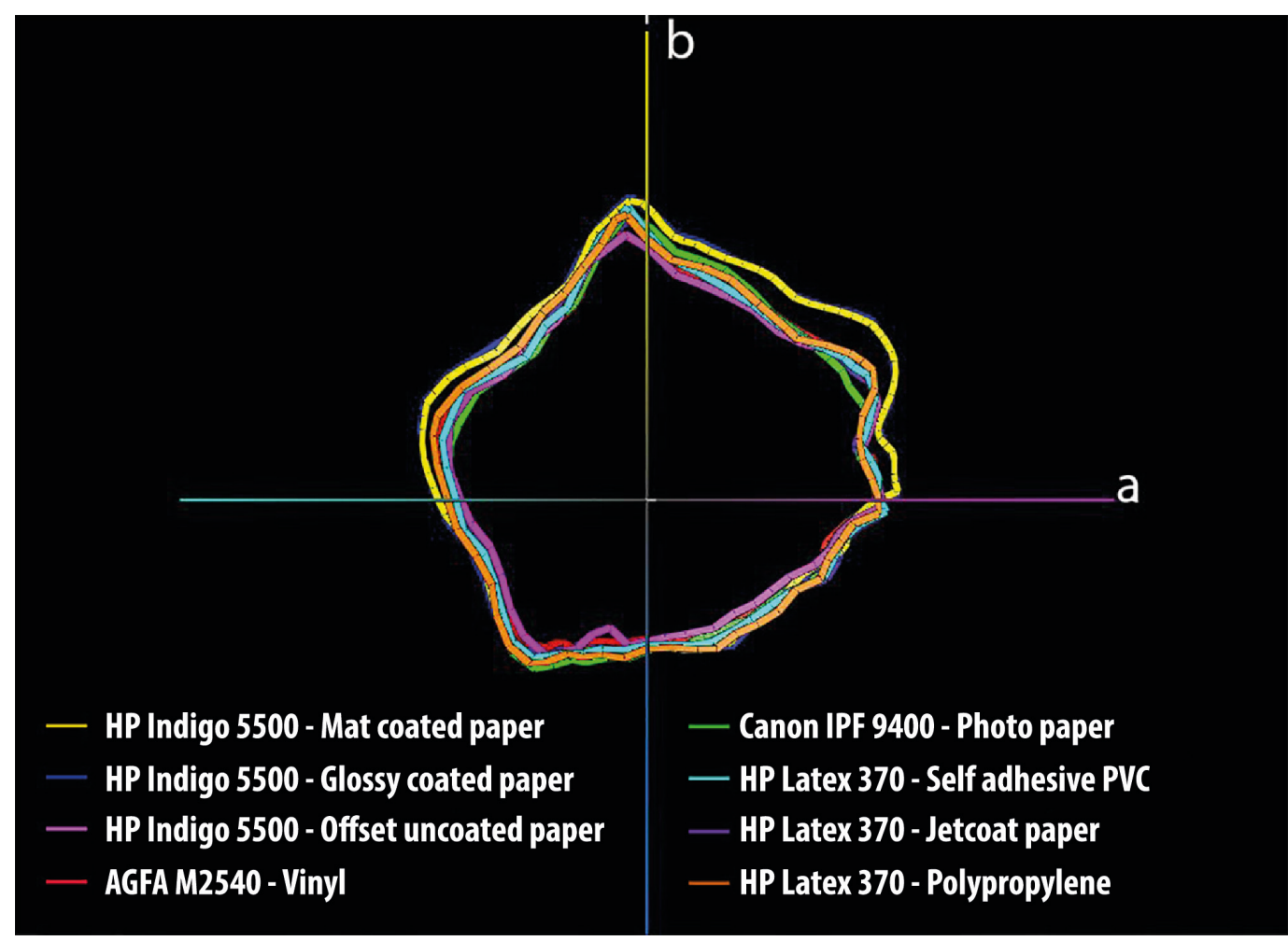

Figure 13: 2D visualisation of the colour range of all types of media, used within the scope of the experiment

\section{CONCLUSIONS}

Based on the results obtained within the scope of this paper, the following conclusions could be presented:

1. Colour reproduction accuracy is an ever more important factor provided by the increasing usage of digital printing systems, especially in the production of packages, labels and other. In spite of the extended colour gamut in most any digital printing system some of the fundamental problems originate from the insufficient colour reproduction accuracy - especially with Pantone. After printing 240 Pantone colours, a colorimetric measurement and appropriate analyses it was established that:

- The average colour difference in ascending order between the set Pantone colours and the measured prints is:
1) $\Delta \mathrm{E}_{00}$ avg. $\mathrm{HP}$ Indigo 5500 varies between 3,83 and 7,06 ( $\Delta \mathrm{E}_{76}$ avg.from 7,59 to 23,33 )
2) $\triangle \mathrm{E}_{00}$ avg. $\mathrm{HP}$ Latex 370 varies between 3,30 and 8,48 ( $\triangle \mathrm{E}_{76}$ avg. from 7,16 to 25,98$)$
3) $\Delta E_{00}$ avg. Canon IPF9400 varies between 4,18 and $7,40\left(\Delta E_{76}\right.$ avg. from 8,28 to 25,37$)$
4) $\Delta E_{00}$ avg. Agfa Anapurna $M 2540$ varies between 3,44 and 8,26 ( $\Delta E_{76}$ avg. from 7,34 to 25,87 )

- The minimal colour difference in ascending order is $\Delta \mathrm{E}_{00}=0,48$ (HP Latex 370) up to 4,10 (HP Indigo 5500)

- The maximal colour difference in ascending order is $\Delta \mathrm{E}_{00}=5,95$ (HP Indigo 5500) up to 26,06 (HP Latex 370)

From the received results one may conclude that the highest colour reproduction accuracy of the Pantone colours is achieved while using the machine HP Indigo 5500 followed by all the other examined printing systems. Therefore, the present paper focuses mainly on its results as closest to ISO12647$2: 2013$. However, the average colour difference $\Delta E$ is relatively high and could be the source of some inaccuracy perception, especially with pretentious customers who often insist on a maximal tolerance of $\pm 1 \div 2 \Delta \mathrm{E}$. For some of the Pantone colours, one receives very good and low values of $\Delta \mathrm{E}$ such as $\Delta \mathrm{E}_{00}=0,48$ (HP Latex 370).

2. Colour gammuts and their volume are of special significance when the print quality is defined. The largest colour gamut was obtained with HP Indigo 5500 ( $\Delta E^{3}=314299$ ), followed from HP Latex 370 
$\left(\Delta E^{3}=254\right.$ 321), Canon IPF9400 $\left(\Delta E^{3}=246\right.$ 780) and Agfa Anapurna M2540 ( $\Delta E^{3}=244$ 498). The standard colour gammuts of FOGRA52 were best met by the HP Indigo 5500, followed by HP Latex 370 and Agfa Anapurna M2540.

3. The tone value increase and colour characteristics of primary colours are important for the research of comparable visual results of digital print compared to the conventional printing processes. The experiment established that the TVI curves of digital printing systems differ significantly from their standardised counterparts. For the most cases, the curves follow the standards for light and dark hues and differ in the mids.

From the presented graphic material, depicting the dependency between the reference and measured colour tone, representing the gradation characteristics, one may observe a uniform increment of the colour tone for the four primary colours with all tested media.

For the most part of the tested media, one observes that within the first $5 \%$ of the reference there is $0 \%$ increase of the colour tone for some of the colours. Another interesting fact, which becomes apparent from these graphics, is that with most any media, the obtained values during the primary colours measurements are slightly lower in the light and mid hues - up to the $60 \%$ mark. On the one hand this does not lead to any significant increase of the colour tone, but the very fact that there are lower values obtained for the CMYK across most of the media could also lead to some inconsistencies in tonal and colour reproduction. From the results obtained herein, one could begin to understand how important it is to adhere as much as possible to the recommendations of the ISO standards in order to be able to optimise and to maintain the print quality so that the customer's highest requirements, with respect to the end product, are fully met. This is especially important with the print of packages, labels and other.

\section{REFERENCES}

[1] Kachin, N., Spiridonov, I.: "Optical Density and Colour Difference in Printing on Different Types of Paper", Cellulose Chemistry And Technology, 39 (3-4), 255-264, 2004.

[2] Kachin, N., Spiridonov I.: "Printing Processes - Part 1, Theoretical bases", (Pleyada, Sofia,2000.)

[3] Kipphan, H.: "Handbook of Print Media", (Springer-Verlag Berlin Heidelberg, Heidelberg, 2001). doi: 10.1007/978-3-540-29900-4

[4] International Organization for Standardization, ISO 12647-2:2013 Graphic technology - Process control for the production of half-tone colour separations, proof and production prints - Part 2: Offset lithographic processes, International Organization for Standardization, 2013.

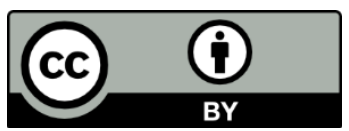

(C) 2018 Authors. Published by the University of Novi Sad, Faculty of Technical Sciences, Department of Graphic Engineering and Design. This article is an open access article distributed under the terms and conditions of the Creative Commons Attribution license 3.0 Serbia (http://creativecommons.org/licenses/by/3.0/rs/). 